\title{
Pars Plana Vitrectomy versus Combined Scleral Buckling-Pars Plana Vitrectomy for Phakic Rhegmatogenous Retinal Detachment with Inferior Breaks
}

\author{
Bhuvan Chanana, Raj Azad \\ University of Delhi, New Delhi, India \\ Email: bhuvan_chanana@rediffmail.com
}

Received 18 April 2016; accepted 24 June 2016; published 27 June 2016

Copyright (C) 2016 by authors and Scientific Research Publishing Inc.

This work is licensed under the Creative Commons Attribution International License (CC BY). http://creativecommons.org/licenses/by/4.0/

(c) (i) Open Access

\begin{abstract}
Aims: To compare the results of pars plana vitrectomy (PPV) and combined scleral buckling-PPV (SB/PPV) in phakic rhegmatogenous retinal detachments with inferior breaks. Methods: Randomized, prospective, clinical controlled trial of forty consecutive phakic eyes with primary rhegmatogenous retinal detachment, associated with inferior breaks and not complicated by proliferative vitreoretinopathy $\geq$ grade C, to either PPV (group 1) or combined SB/PPV (group 2). Results: At 6 months follow up the primary reattachment rate was $100 \%(20 / 20$ cases) in group 2 and $70 \%$ $(14 / 20$ cases $)$ in the group 1 , the difference being statistically significant $(p=0.027)$. The best corrected visual acuity improved significantly from a preoperative mean of $1.65 \pm 1.13$ (Range: 0.6 to 3 ) to a mean of $0.45 \pm 0.11$ (Range: 0.3 to 0.6 ) in the group 2 and in the group 1 improved from a preoperative mean of $2.34 \pm 0.92$ (Range: 0.48 to 3 ) to a mean of $0.668 \pm 0.20$ (Range: 0.48 to 1 ), the difference between the two groups being statistically significant $(p=0.001)$. Conclusion: Anatomical and functional success rates are significantly better with the use of a scleral explant during PPV for uncomplicated forms of phakic rhegmatogenous retinal detachments with inferior breaks.
\end{abstract}

\section{Keywords}

Pars Plana Vitrectomy, Proliferative Vitreoretinopathy, Rhegmatogenous Retinal Detachment, Scleral Buckling 


\section{Introduction}

Rhegmatogenous retinal detachment (RRD) is the separation of the neurosensory retina from the underlying retinal pigment epithelium. Vitreoretinal traction is responsible for the occurrence of most RRD. As the vitreous becomes more syneretic (liquefied) with age, a posterior vitreous detachment (PVD) occurs. In certain eyes, strong vitreoretinal adhesions are present and the occurrence of a PVD can lead to a retinal tear formation; then, fluid from the liquefied vitreous can seep under the tear, leading to a retinal detachment.

The use of pars plana vitrectomy (PPV) in the treatment of rhegmatogenous retinal detachment has gained increasing popularity over the past 20 years [1]-[4]. However, inferior breaks present a surgical challenge as it is recognized that intraocular tamponade by gas or silicone oil is unable to provide direct support to the inferior breaks. A scleral buckle (SB) during PPV is used to produce an inferior indent to support to the inferior retina [2] [5]. However, the use of scleral explants with PPV is associated with several risks including hypotony during placement of the buckle with associated choroidal hemorrhage [6]. Postoperative complications include extrusion, intrusion or infection of exoplant material [7], motility problems [8], refractive changes [9], diplopia [8] [10], and anterior segment ischemia [11].

Primary vitrectomy alone is an attractive alternative in these cases because it affords a direct approach to vitreous traction and avoids the complication associated with a scleral explant [12]. A few studies in recent past have reported that a supplementary SB, to support the inferior retina is unnecessary and good results are achieved with pars plana vitrectomy alone in RRD with inferior breaks [13] [14].

We undertook this study to determine whether scleral explants are required during pars plana vitrectomy in the management of RRD with inferior breaks in phakic eyes, not complicated by PVR $\geq$ grade C. The study is a randomized prospective comparison of primary vitrectomy and combined scleral buckling-pars plana vitrectomy (SB/PPV), with respect to the primary and final anatomic and functional success rates and the rates of complications. The goal of the clinical trial was the improvement of surgical therapy of phakic RRD associated with inferior breaks.

\section{Materials and Methods}

In this study, an open-label randomized prospective comparison of pars plana vitrectomy and combined SB/PPV was carried out in phakic RRD with inferior breaks. Forty consecutive phakic eyes with primary rhegmatogenous retinal detachment and inferior retinal breaks anterior to the equator between 4 and 8 o'clock, not complicated by proliferative vitreoretinopathy grade $\mathrm{C}$ or greater, and having a clear lens, were included in the study. The study had to be completed within a specified period of 3 years and the sample size was one of logistical feasibility. The patients were selected from vitreo-retina services of our institute from August 2010 to July 2013. All cases having proliferative vitreoretinopathy (PVR) grade C or more, no break retinal detachment, aphakia or pseudophakia, giant retinal tear and history of any previous intra-ocular surgery were excluded from the study.

Prior approval was obtained from the Ethics Committee of All India Institute of Medical Sciences, New Delhi and the study was in accordance with declaration of Helsinki. All the patients were drawn from the vitreoretina services of University College of Medical Sciences. An informed consent was obtained from all the patients prior to the surgical procedure.

All patients were randomly assigned to one of the two treatment arms with 20 eyes in each group. Each patient was given one of a set of sequentially numbered randomization envelopes containing one of the two surgical options; the patient then received the treatment detailed in the envelope.

All surgical procedures were performed under local peribulbar anaesthesia by one surgeon (BC). When combined scleral buckling-vitrectomy was performed, a 360 degree exoplant (segmental $7 \mathrm{~mm}$ silicone tire) was used to support the inferior retinal breaks. All eyes underwent a three-port 20 gauge pars plana vitrectomy, relieving the vitreous traction around the break and removal of accessible vitreous using wide-angle lenses and scleral indentation. Posterior vitreous detachment was induced when not already present with the help of Triamcinolone Acetate and vitrectomy completed. This was followed by fluid-air exchange with internal drainage of subretinal fluid either through break or retinotomy. Retinopexy was achieved by diode laser endophotocoagulation (360 degree along with delineation of the break and retinotomy). An air-silicone oil (5000 cS silicone oil) exchange was carried out to achieve a complete fill of the vitreous cavity.

Patients of both groups were instructed for head positioning to encourage tamponade of the retinal breaks during the first two postoperative weeks. Topical antibiotics, anti-inflammatory and cycloplegic drops were ap- 
plied postoperatively for 1 month, according to our department's protocol. Silicone oil removal was carried out at 3 months, and combined with phacoemulsification and lens implantation if cataract developed. All eyes were evaluated on day 7, at 1 month, 3 months and 6 months of follow up. The parameters evaluated included best corrected visual acuity (BCVA), IOP (always measured by applanation tonometry); lens status; and fundus examination. Patients with redetachment were operated again with silicone oil tamponade and causes of surgical failure were noted. An explant was not used in cases undergoing re-surgery. Postoperative complications were also recorded. Only patients who completed 6 months of follow up were included in the final analysis.

\section{Statistical Analysis}

Contingency tables were drawn for the variables in the two groups and analyzed using the Pearson's $\chi^{2}$ test. For continuous variables such as the visual acuity in logMAR and IOP, Student's $t$-test for statistical significance was used. The statistical analysis was performed on the Statistical Package for the Social Sciences (spss version 10) software (SPSS, Chicago, IL, USA). For statistical significance, $P$ was set at $\leq 0.05$.

Visual acuity was analyzed for study purposes by means of the logarithm of the minimal angle of resolution (logMAR) score, developed by Ferris and associates [15]. Conversion of counting fingers and hand-motion visual acuity to Snellen equivalent was made according to the method proposed by Holladay [16] and were given values of $0.01(+2.0 \log$ MAR) and $0.001(+3.0 \log$ MAR), respectively.

\section{Results}

The pars plana vitrectomy (PPV) and combined scleral buckling-PPV (SB/PPV) groups were similar with respect to the preoperative factors of age, sex, laterality of the eye, duration of the RD, macular status, presence of risk factors, number of breaks, preoperative visual acuity and preoperative IOP, and there were no statistically significant differences between the two groups (Table 1). All cases were followed up for a minimum period of 6 months.

Table 1. Basic characteristics of patients in the 2 treatment groups at the beginning of study.

\begin{tabular}{|c|c|c|c|}
\hline & PPV group $(n=20)$ & SB/PPV group $(n=20)$ & $P$ value \\
\hline Age mean \pm SD & $27 \pm 10$ years & $28 \pm 11$ years & 0.624 \\
\hline \multicolumn{4}{|l|}{$\operatorname{Sex}(\%[n])$} \\
\hline Male & $60 \%(\mathrm{n}=12)$ & $40 \%(n=8)$ & 0.206 \\
\hline Female & $40 \%(n=8)$ & $60 \%(n=12)$ & \\
\hline Laterality of eye (right eye-\% [no.]) & $55 \%(\mathrm{n}=11)$ & $50 \%(n=10)$ & 0.523 \\
\hline Duration of RD (mean [Range]) & 27 days (15 - 60 days) & 27 days (10 - 45 days) & 0.968 \\
\hline Macula off (\% [no.]) & $100 \%(n=20)$ & $100 \%(n=20)$ & 1.00 \\
\hline LogMAR (BCVA mean \pm SD) & $2.348 \pm 0.923$ & $1.654 \pm 1.133$ & 0.096 \\
\hline \multicolumn{4}{|l|}{ Risk factors present (\% [no.]) } \\
\hline Myopia (>-6D) & $20 \%(n=4)$ & $30 \%(n=6)$ & 0.454 \\
\hline Peripheral degeneration & $35 \%(n=7)$ & $20 \%(n=4)$ & \\
\hline PVD & $15 \%(n=3)$ & $30 \%(n=6)$ & \\
\hline Trauma & $30 \%(n=6)$ & $20 \%(n=4)$ & \\
\hline Preoperative IOP, mm Hg (mean \pm SD) & $12 \pm 2$ & $12.2 \pm 1.6$ & 0.731 \\
\hline \multicolumn{4}{|l|}{ Number of breaks } \\
\hline 1 & $40 \%(n=8)$ & $30 \%(n=6)$ & 0.776 \\
\hline 2 & $20 \%(n=4)$ & $20 \%(n=4)$ & \\
\hline 3 or more & $40 \%(n=8)$ & $50 \%(n=10)$ & \\
\hline
\end{tabular}

BCVA = best-corrected visual acuity; IOP = intraocular pressure; LogMAR = logarithm of minimum angle of resolution; PVD = posterior vitreous detachment; $\mathrm{RD}=$ retinal detachment. 
A primary anatomical success rate (Table 2 ) of 70\% (14/20 cases) was achieved in the PPV group compared to $100 \%$ (20/20 cases) in the SB/PPV group, the difference between the two groups being statistically significant $(p=0.027)$. At 7 days no redetachment was observed in either group. Redetachment of the retina was observed in 2 eyes at 1 month and 4 eyes at 3 months follow up in the PPV group. An assessment of the cause for surgical failures in the PPV group showed open breaks (new or missed breaks) in two cases, PVR in three cases and both PVR and open breaks in one case. The final anatomical success rate at the end of 6 months was $100 \%$ in both the groups.

The difference in the final best corrected visual acuity (BCVA) achieved in the two groups were statistically significant $(\mathrm{p}=0.001)$. The logMAR BCVA in the PPV group improved from a preoperative mean of $2.34 \pm$ 0.92 (Range: 0.48 to 3 ) to a mean of $0.87 \pm 0.81$ at 1 month and $0.92 \pm 0.23$ at 3 months. The final logMAR BCVA in the PPV group improved to a mean of $0.668 \pm 0.20$ (Range: 0.48 to 1). Eight eyes (40\%) achieved vision $\geq 0.6(6 / 24)$, whereas only one eye (5\%) showed a drop of $\geq 1$ line of Snellen acuity. Two eyes (10\%) maintained their preoperative visual acuity. In the SB/PPV the logMAR BCVA improved from a preoperative mean of $1.65 \pm 1.13$ (Range: 0.6 to 3 ) to a mean of $0.57 \pm 0.31$ at 1 month and $0.51 \pm 0.29$ at 3 months. The final $\operatorname{logMAR}$ BCVA improved to a mean of $0.45 \pm 0.11$ (Range: 0.3 to 0.6 ). Sixteen eyes $(80 \%$ ) achieved vision $\geq$ 0.6 (6/24), while none of the eyes showed a drop of $\geq 1$ line of Snellen acuity. One eye (5\%) maintained its preoperative visual acuity.

The major intra-operative and post-operative complications encountered during the study are summarized in Table 3. Iatrogenic breaks occurred in four cases (20\%) in the SB/PPV group and in three cases (15\%) in the PPV group, while completing the vitreous base dissection; the breaks were delimited by laser photocoagulation. Postoperatively in the SB/PPV group two cases (10\%) had raised IOP (defined as IOP > $21 \mathrm{mmHg}$ on at least two occasions in the first postoperative week) which was controlled on medical management. Buckle infection

Table 2. Anatomical and functional outcomes observed in the 2 groups.

\begin{tabular}{ccc}
\hline Variable & Vitrectomy group & Combined group \\
\hline \% surgical primary success & $70 \%(14 / 20)$ & $\begin{array}{c}100 \%(20 / 20) \\
(\mathrm{p}=0.027)\end{array}$ \\
& $\begin{array}{c}\text { 2 cases: open break } \\
\text { 3 cases: PVR }\end{array}$ & \\
Cause of failure & 1 case: open break + PVR & \\
Final visual acuity & Mean: $0.668 \pm 0.204$ & Mean: $0.45 \pm 0.111$ \\
& Range: 0.48 to 1.0 & Range: 0.3 to 0.6 \\
\hline
\end{tabular}

Table 3. Complications observed in the 2 groups.

\begin{tabular}{ll}
\hline \multicolumn{1}{c}{ Complications } & No. of cases \\
\hline Vitrectomy group & $3(15 \%)$ \\
Iatrogenic breaks & $6(30 \%)$ \\
Cataract & $2(10 \%)$ \\
Raised intraocular pressure & $1(5 \%)$ \\
Persistent epithelial defect & \\
Combined group & $4(20 \%)$ \\
Iatrogenic breaks & $2(10 \%)$ \\
Cataract & $2(10 \%)$ \\
Raised intraocular pressure & $1(5 \%)$ \\
Epiretinal membrane & $1(5 \%)$ \\
Buckle infection & \\
\hline
\end{tabular}


was seen in one case (5\%) and one case (5\%) developed epiretinal membrane (ERM). In the PPV group there was development of cataract in six cases (30\%), while only two cases (10\%) developed cataract in the SB/PPV group; the difference between the two groups was not statistically significant at $\mathrm{p}=0.120$. Raised IOP was present in two cases $(10 \%)$ which were controlled on medical management and one case developed a persistent epithelial defect which healed with the use of a bandage contact lens.

\section{Discussion}

We conducted a randomized, prospective, controlled clinical trial comparing pars plana vitrectomy and combined scleral buckling-pars plana vitrectomy with respect to the anatomical and functional outcomes in primary rhegmatogenous retinal detachment with inferior breaks complicated by PVR $<$ grade $C$ in phakic eyes. Forty cases of primary rhegmatogenous retinal detachment, complicated by PVR < grade C, with a clear media were selected from vitreoretina services of our institute from August 2010 to July 2013. All eyes were screened meticulously with indirect ophthalmoscopy and fundus biomicroscopy using scleral indentation preoperatively. Post-operatively all the cases were followed up for a minimum period of 6 months as per the study protocol. All the analysis and results pertain to the outcome at the 6 months follow up. Although studies showing good results with primary vitrectomy alone in RRD with inferior breaks have been done [13] [14], this is the first randomized prospective study in which pars plana vitrectomy for uncomplicated RRD with inferior breaks in phakic eyes is being compared with combined scleral buckling and pars plana vitrectomy.

In our series, the differences in the primary anatomical and functional success rates between the two groups were statistically significant. The final attachment rate was however $100 \%$ in both the groups at the end of 6 months follow up. Some studies in the past have shown that adequate success rates can be achieved when repairing RRD, with inferior breaks, using vitrectomy without scleral buckling [13] [14]. The primary success rate for RRD with inferior breaks achieved by sharma et al. [14] was $81.3 \%$ and final success rates were $95.8 \%$. However in our study, a 360 degree scleral buckle during PPV to support the inferior breaks, achieved superior results. Although segmental buckle is sufficient to support retinal break, a 360 degree buckle explant was used not only to combat the retinal break but also to combat subsequent development of PVR and retinal shortening.

It may be that RRD with inferior breaks performs less well because it is more prone to the effects of PVR, which is more likely to occur inferiorly. One of the inherent problems in the use of PPV is the difficulty of producing a direct tamponade on inferior retinal breaks using currently available intraocular tamponade agents [5] [17]. Perfluorocarbon liquids have been used on a short term basis for postoperative tamponade of inferior breaks but are associated with retinal toxicity, an increased risk of PVR [18] and require further intervention to ensure removal from the eye [19]. In our study we used silicone oil (5000 cS silicone oil), since it provides better tamponade for inferior breaks compared with intraocular gases. With inferior breaks and gas, post-operative positioning is very important to tamponade the break. Patient compliance could not be relied upon, and hence, to be on the safe side, silicone oil was used. High-density silicone oil (HDSO) has been used as an internal retinal tamponade for complicated retinal detachments [20] [21]. HDSO are associated with subretinal proliferations and early emulsification [21]. The Heavy Silicone Oil versus Standard Silicone Oil Study (HSO study) results failed to demonstrate superiority of a heavy tamponade [20].

Although several authors have addressed the issue of PPV for RRD few have specifically investigated the management of inferior retinal breaks and the use of supplementary scleral buckles. Gartry et al. [2] identified eight eyes with primary $\mathrm{RD}$ associated with inferior retinal breaks in a mixed series of 114 eyes undergoing PPV. They treated these cases with a supplementary inferior segmental buckle and achieved $75 \%$ (6/8) success with one operation. Heimann et al. [22] in a retrospective series of 53 patients identified six patients with primary RD associated with inferior retinal breaks which they treated with PPV and SF6 tamponade without scleral buckle. Retinal redetachment occurred in 50\% (3/6) of these eyes. Campo et al. [23] reported on 283 consecutive pseudophakic eyes undergoing PPV without the use of scleral buckling to repair RD. They reported an $88 \%$ reattachment rate with one operation that included gas tamponade and 360 degree prophylactic laser. No information was provided on the position of retinal breaks.

Over the years PPV has been used without scleral buckling for the management of RRD [12] [24] [25]. We have also conducted similar studies at our centre, evaluating the role of primary vitrectomy in phakic retinal detachments [24] and pseudophakic retinal detachments [25] with satisfactory results. But inferior breaks have rarely have received specific attention as a separate group in all these studies. 
Visually significant cataract requiring extraction developed in 6 eyes (30\%) in the PPV group. Cataract formation has been recorded as a complication of vitrectomy in many studies [4] [22] [26]. In the SB/PPV group 2 eyes (10\%) developed visually significant cataract requiring lens extraction at the end of 6 months. The difference was not statistically significant, however it would be inappropriate to comment on difference in the occurrence of cataract, if any, unless the follow-up period is quite long.

With the introduction of wide angle viewing systems, although maximum removal of vitreous gel is possible during PPV, but in phakic eyes it is difficult to complete vitrectomy anteriorly and it is fraught with the danger of injuring the lens. A scleral buckle indents the retina inwards and facilitates anterior vitrectomy preventing the risk of lens trauma. This could possibly explain the lower number of visually significant cataract in the SB/PPV group. However we would expect to see more cataract development in both groups with a longer duration of follow up.

Raised IOP was present in 2 cases each from both groups. The raised IOP was transient in both groups and controlled by topical antiglaucoma medications or systemic carbonic anhydrase inhibitors, with no serious complications. Elevated IOP is a known complication following frequent fluid-gas exchange in vitrectomy [27].

Our study had some limitations. The duration of the two operative procedures were not compared. Also the levels of patient discomfort during surgery and postoperatively were also not compared. Scleral buckling is expected to give more intraoperative and postoperative discomfort (pain, redness, lid edema). A discomfort scale designed to include all these factors and applied to test both procedures can help elucidate this problem. The sample size of the study was also small.

The strong points in favor of this study are its prospective nature and randomization of consecutive patients, and the fact that possible confounding factors were either excluded (giant retinal tears, unseen breaks) or found not to differ significantly between the two groups (age, duration of the RD, macular status, presence of risk factors, preoperative visual acuity).

\section{Conclusion}

To conclude, in this randomized clinical trial, we found that with primary vitrectomy combined with a scleral explant, a high final anatomical and functional success rate can be achieved in uncomplicated forms of phakic rhegmatogenous retinal detachments associated with inferior breaks. In view of the above, we recommend scleral buckling during vitrectomy as the primary surgical modality in the treatment of uncomplicated, phakic rhegmatogenous retinal detachments where inferior breaks are present. However, a larger multicentre trial may be required to consolidate the results.

\section{References}

[1] Ah-Fat, F.G., Sharma, M.C., Majid, M.A., McGalliard, J.N. and Wong, D. (1999) Trends in Vitreoretinal Surgery at a Tertiary Referral Centre: 1987 to 1996. British Journal of Ophthalmology, 83, 396-398. http://dx.doi.org/10.1136/bjo.83.4.396

[2] Gartry, D.S., Chignell, A.H., Franks, W.A. and Wong, D. (1993) Pars Plana Vitrectomy for the Treatment of Rhegmatogenous Retinal Detachment Uncomplicated by Advanced Proliferative Vitreoretinopathy. British Journal of Ophthalmology, 77, 199-203. http://dx.doi.org/10.1136/bjo.77.4.199

[3] Leaver, P. (1993) Expanding the Role of Vitrectomy in Retinal Reattachment Surgery. British Journal of Ophthalmology, 77, 197. http://dx.doi.org/10.1136/bjo.77.4.197

[4] Hakin, K.N., Lavin, M.J. and Leaver, P.K. (1993) Primary Vitrectomy for Rhegmatogenous Retinal Detachment. Graefe's Archive for Clinical and Experimental Ophthalmology, 231, 344-346. http://dx.doi.org/10.1007/BF00919031

[5] Fawcett, I.M., Williams, R.L. and Wong, D. (1994) Contact Angles of Substances Used for Internal Tamponade in Retinal Detachment Surgery. Graefe's Archive for Clinical and Experimental Ophthalmology, 232, 438-444. http://dx.doi.org/10.1007/BF00186587

[6] Tabandeh, H., Sullivan, P.M., Smahliuk, P., Flynn Jr., H.W. and Schiffman, J. (1999) Suprachoroidal Hemorrhage during Pars Plana Vitrectomy. Risk Factors and “Outcomes”. Ophthalmology, 106, 236-242. http://dx.doi.org/10.1016/S0161-6420(99)90062-3

[7] Flindall, R.J., Norton, E.W., Curtin, V.T. and Gass, J.D. (1971) Reduction of Extrusion and Infection Following Episcleral Silicone Implants and Cryopexy in Retinal Detachment Surgery. American Journal of Ophthalmology, 71, 835837. http://dx.doi.org/10.1016/0002-9394(71)90250-9

[8] Arruga, A. (1977) Motility Disturbances Induced by Operations for Retinal Detachment. Modern Problems in Oph- 
thalmology, 18, 408-414.

[9] Goel, R., Crewdson, J. and Chignell, A.H. (1983) Astigmatism Following Retinal Detachment Surgery. British Journal of Ophthalmology, 67, 327-329. http://dx.doi.org/10.1136/bjo.67.5.327

[10] Fison, P.N. and Chignell, A.H. (1987) Diplopia after Retinal Detachment Surgery. British Journal of Ophthalmology, 71, 521-525. http://dx.doi.org/10.1136/bjo.71.7.521

[11] Kwartz, J., Charles, S., McCormack, P., Jackson, A. and Lavin, M. (1994) Anterior Segment Ischaemia Following Segmental Scleral Buckling. British Journal of Ophthalmology, 78, 409-410. http://dx.doi.org/10.1136/bjo.78.5.409

[12] Escoffery, R.F., Olk, R.J., Grand, M.G. and Boniuk, I. (1985) Vitrectomy without Scleral Buckling for Primary Rhegmatogenous Retinal Detachment. American Journal of Ophthalmology, 99, 275-281. http://dx.doi.org/10.1016/0002-9394(85)90356-3

[13] Tanner, V., Minihan, M. and Williamson, T.H. (2001) Management of Inferior Breaks during Pars Plana Vitrectomy for Retinal Detachement. British Journal of Ophthalmology, 85, 480-482. http://dx.doi.org/10.1136/bjo.85.4.480

[14] Sharma, A., Grigoropoulas, V. and Williamson, T.H. (2004) Management of Primary Rhegmatogenous Retinal Detachment with Inferior Breaks. British Journal of Ophthalmology, 88, 1372-1375. http://dx.doi.org/10.1136/bjo.2003.041350

[15] Ferris, F.L., Kassoff, A., Bresnick, G.H. and Bailey, I. (1982) New Visual Acuity Charts for Clinical Research. American Journal of Ophthalmology, 94, 91-96. http://dx.doi.org/10.1016/0002-9394(82)90197-0

[16] Holladay, J.T. (1997) Proper Method for Calculating Average Visual Acuity. Journal of Refractive Surgery, 13, 388391.

[17] Williams, R. and Wong, D. (1999) The Influence of Explants on the Physical Efficiency of Tamponade Agents. Graefe's Archive for Clinical and Experimental Ophthalmology, 237, 870-874. http://dx.doi.org/10.1007/s004170050325

[18] De Molfetta, V., Bottoni, F., Arpa, P., Vinciguerra, P. and Zenoni, S. (1992) The Effect of Simultaneous Internal Tamponade on Fluid Compartmentalization and Its Relationship to Cell Proliferation. Retina, 12, S40-S45. http://dx.doi.org/10.1097/00006982-199212031-00009

[19] Bottoni, F., Sborgia, M., Arpa, P., De Casa, N., Bertazzi, E., Monticelli, M. and De Molfetta, V. (1993) Perfluorocarbon Liquids as Postoperative Short-Term Vitreous Substitutes in Complicated Retinal Detachment. Graefe's Archive for Clinical and Experimental Ophthalmology, 231, 619-628. http://dx.doi.org/10.1007/BF00921955

[20] Joussen, A.M., Rizzo, S., Kirchhof, B., Schrage, N., Li, X., Lente, C. and Hilgers, R.D. (2011) HSO-Study Group: Heavy Silicone Oil versus Standard Silicone Oil in as Vitreous Tamponade in Inferior PVR (HSO Study): Interim Analysis. Acta Ophthalmologica, 89, e483-e489. http://dx.doi.org/10.1111/j.1755-3768.2011.02139.x

[21] Ozdek, S., Yuksel, N., Gurelik, G. and Hasanreisoglu, B. (2011) High-Density Silicone Oil as an Intraocular Tamponade in Complex Retinal Detachments. Canadian Journal of Ophthalmology, 46, 51-55. http://dx.doi.org/10.3129/i10-107

[22] Heimann, H., Bornfeld, N., Friedrichs, W., Helbig, H., Kellner, U., Korra, A. and Foerster, M.H. (1996) Primary Vitrectomy without Scleral Buckling for Rhegmatogenous Retinal Detachment. Graefe's Archive for Clinical and Experimental Ophthalmology, 234, 561-568. http://dx.doi.org/10.1007/BF00448800

[23] Campo, R.V., Sipperley, J.O., Sneed, S.R., Park, D.W., Dugel, P.U., Jacobsen, J. and Flindall, R.J. (1999) Pars Plana Vitrectomy without Scleral Buckle for Pseudophakic Retinal Detachments. Ophthalmology, 106, 1811-1815. http://dx.doi.org/10.1016/S0161-6420(99)90353-6

[24] Azad, R.V., Chanana, B., Sharma, Y.R. and Vohra, R. (2007) Primary Vitrectomy versus Conventional Retinal Detachment Surgery (PCR Study), in Phakic Rhegmatogenous Retinal Detachments. Acta Ophthalmologica Scandinavica, 85, 540-545. http://dx.doi.org/10.1111/j.1755-3768.2007.00888.x

[25] Sharma, Y.R., Karunanithi, S., Azad, R.V., Vohra, R., Pal, N., Singh, D.V. and Chandra, P. (2005) Functional and Anatomic Outcome of Scleral Buckling versus Primary Vitrectomy in Pseudophakic Retinal Detachment. Acta Ophthalmologica Scandinavica, 83, 293-297. http://dx.doi.org/10.1111/j.1600-0420.2005.00461.X

[26] Oshima, Y., Yamanishi, S., Sawa, M., Motokura, M., Harino, S. and Emi, K. (2000) Two-Year Follow-Up Study Comparing Primary Vitrectomy with Scleral Buckling for Macula-Off Rhegmatogenous Retinal Detachment. Japanese Journal of Ophthalmology, 44, 538-549. http://dx.doi.org/10.1016/S0021-5155(00)00205-7

[27] Bartz-Schmidt, K.U., Kirchhof, B. and Heimann, K. (1996) Primary Vitrectomy for Pseudophakic Retinal Detachment. British Journal of Ophthalmology, 80, 346-349. http://dx.doi.org/10.1136/bjo.80.4.346 


\section{Submit or recommend next manuscript to SCIRP and we will provide best service for you:}

Accepting pre-submission inquiries through Email, Facebook, Linkedin, Twitter, etc A wide selection of journals (inclusive of 9 subjects, more than 200 journals)

Providing a 24-hour high-quality service

User-friendly online submission system

Fair and swift peer-review system

Efficient typesetting and proofreading procedure

Display of the result of downloads and visits, as well as the number of cited articles

Maximum dissemination of your research work

Submit your manuscript at: http://papersubmission.scirp.org/ 